\title{
Índices de depressão, ansiedade e estresse entre estudantes de enfermagem e medicina do Acre
}

\author{
Rates of depression, anxiety and stress among \\ nursing students and medicine of Acre
}

\section{Mathews Barbosa Santiago' 1 Odete Silva Braga² (1) \\ Polyanna Rodrigues da Silva ${ }^{3}$ (1) Vinicius Matheus Ritter Capelli ${ }^{4}(1)$ Ruth Silva Lima da Costa ${ }^{5}$}

\author{
1-4Centro Universitário UNINORTE (Rio Branco). Acre, Brasil. mathewsbarbosa@hotmail.com, odethebraga14@hotmail.com, \\ polyanna.xapuri@gmail.com, viniciusuninorte@gmail.com \\ ${ }^{5}$ Autora para correspondência. Centro Universitário UNINORTE (Rio Branco). Acre, Brasil. ruttylima@hotmail.com
}

\begin{abstract}
RESUMO | INTRODUÇÃo: Atualmente existe uma grande preocupação da sociedade, quanto ao aumento do número de estudantes com sintomas de depressão, ansiedade e estresse, principalmente dentro das universidades, devido a vários fatores e dentre eles destacam-se a sobrecarga de estudos e a necessidade de adoção de um novo estilo de vida. OBJETIVOS: O presente artigo buscou evidenciar índices de depressão, ansiedade e estresse entre estudantes dos cursos de enfermagem e medicina de um centro universitário do Acre. MÉTODOS: Trata-se de um estudo transversal, descritivo e de abordagem quantitativa, desenvolvido junto a 80 alunos dos cursos de medicina e enfermagem. RESULTADOS: A maioria dos estudantes pertenciam ao sexo feminino, eram solteiros, procedentes do Acre, residiam com outras pessoas, nunca tinham realizado psicoterapia e nem tratamento psiquiátrico. Também não faziam uso de medicamentos para melhorar o desempenho acadêmico. Quanto as médias de sintomas de depressão, ansiedade e estresse entre os acadêmicos avaliados, os do curso de enfermagem apresentaram a maior média de sintomas de depressão e ansiedade e os do curso de medicina mais sintomas de estresse. Quando comparados por semestres, os estudantes do primeiro semestre de enfermagem apresentaram mais sintomas depressão e ansiedade e os do oitavo semestre de medicina, apresentaram mais estresse. CONCLUSÃo: A saúde mental dos estudantes pode encontra-se em risco, o que evidencia a necessidade de implementação de um projeto político-pedagógico pela instituição de ensino, voltado para essa temática, apresentando estratégias para o enfrentamento dessa realidade, que vise o bem-estar dessa população.
\end{abstract}

PALAVRAS-CHAVE: Saúde Mental. Estresse psicológico. Ansiedade. Depressão.
ABSTRACT | INTRODUCTION: Currently there is a great concern of society, regarding the increase in the number of students with symptoms of depression, anxiety, and stress, especially within universities, due to several factors and among them stand out the overload of studies and the need for the adoption of a new lifestyle. OBJECTIVE: This article aimed to evidence rates of depression, anxiety, and stress among students of nursing and medical courses in a university center in Acre. METHODS: This is a cross-sectional, descriptive, and quantitative study developed with 80 students of medical and nursing courses. RESULTS: Most of the students were female, were single, from Acre, lived with other people, had never undergone psychotherapy or psychiatric treatment. Also, they did not use medications to improve academic performance. Concerning the mean symptoms of depression, anxiety, and stress among the students evaluated, those in the nursing course had the highest mean symptoms of depression and anxiety and those in the medical course plus stress symptoms. When compared by semesters, the students of the first semester of nursing presented more symptoms of depression and anxiety and those in the eighth semester of medicine presented more stress. CONCLUSIONS: The mental health of students may be at risk, which evidences the need to implement a political-pedagogical project by the educational institution, focused on this theme, presenting strategies to face this reality, aimed at the wellbeing of this population.

KEYWORDS: Mental Health. Psychological stress. Anxiety. Depression. 


\section{Introdução}

Durante a graduação, especialmente nos cursos da área da saúde, os estudantes estão suscetíveis a experiências estressantes, como a exigência de um bom desempenho acadêmico, o contato com pacientes com doenças graves e em estado terminais, extensas horas dedicadas aos estudos, estágios curriculares obrigatórios, plantões, dentre outros, o que pode levar a uma infinidade de manifestações, como o surgimento de sintomas de sofrimento psíquico (Deperon et al., 2013).

Entre os sintomas de sofrimento psíquico, podem estar a depressão, que é uma síndrome caracterizada por manifestações de desinteresse pela vida e pelo trabalho, tristeza sem motivo justificável, desânimo, irritabilidade e insônia. O sentimento de vazio de falta de sentido na vida e de esgotamento caracterizam os casos mais graves, chegando à ideia e tentativas de suicídio (Jardim, 2011).

Nesse momento da vida acadêmica, os estudantes também podem manifestar sintomas de ansiedade, que pode ser caracterizada como uma emoção própria da existência humana, sendo considerada uma reação natural, mais que pode gerar sensações de apreensão e alterações físicas desagradáveis frente a situações de desafiadoras e até mesmo desconhecidas (Claudino; Cordeiro, 2016).

A ansiedade apresenta-se como um problema constante para muitos estudantes, pois, a pressão exercida para a resolutividade das pendências exigidas pela academia, transforma a experiência do ensino superior cada vez mais problemática, levando-os em alguns casos, a desenvolverem doenças psicopatológicas, por conta do seu estado emocional (Leão, 2018).

Outro sintoma muito comum entre os estudantes é o estresse, que pode ser definido como uma reação natural do organismo, que ocorre mediante a situações de perigo ou ameaça, provocando alterações físicas e emocionais (Balone, 2020), reação essa muito comum entre eles, devido às inúmeras demandas exigidas pela vida acadêmica.
De acordo com dados do relatório do perfil socioeconômico e cultural dos estudantes de graduação das universidades federais brasileiras - Fornaprace (2011), o percentual de estudantes encontrados com queixas de sofrimento psíquico foi de $47,7 \%$, dentre estes, 29\% procuraram atendimento psicológico, $9 \%$ procuraram atendimento psiquiátrico, $11 \%$ usaram ou estavam fazendo o uso de medicação psiquiátrica e $10 \%$ procuraram atendimento psicopedagógico. 0 relatório revelou ainda uma grande parcela dos estudantes queixando-se de dificuldades de adaptação a novas situações, como à cidade, à moradia ou à separação da família.

Estudos têm evidenciado que os índices de depressão, ansiedade e estresse vêm aumentando principalmente entre estudantes dos cursos da área de saúde (Leão et al., 2018; Costa, et al., 2020).

Destarte, é importante frisar que a partir do desenvolvimento dos sintomas de depressão, ansiedade e estresse, os estudantes ficam susceptíveis à utilização de substâncias psicoestimulantes. No estudo de Santos Pires et al. (2018), realizado com 278 alunos regularmente matriculados no curso de medicina, aponta-se que o uso dessas substâncias foi referido por $52,94 \%$, onde o uso de metilfenidato foi citado por $29,9 \%$ dos alunos. Sendo que $66,6 \%$ começaram a utilizar logo após o ingresso no curso. Destes, $88,1 \%$ admitiram que a utilização destas substâncias acontecia às vésperas das provas.

Mediante a isso, torna-se necessário conhecer a realidade dos acadêmicos, identificar tais sintomas e encaminhá-los a serviços de apoio, afim de proporcionar uma melhor qualidade de vida e certamente um melhor rendimento escolar (Alves, 2014).

Levando-se em conta que o dia a dia dentro das universidades requer do estudante a capacidade de adaptar-se às mudanças impostas pela sua nova rotina, a exemplo da carga excessiva de estudos, pressão na realização de trabalhos e provas, desenvolvimento de novas habilidades, dentre outros, podendo gerar nos estudantes sintomas de sofrimento psíquico, o presente estudo buscou evidenciar índices de depressão, ansiedade e estresse entre estudantes dos cursos de enfermagem e medicina de um centro universitário do Acre. 


\section{Métodos}

Trata-se de um estudo transversal, descritivo e de abordagem quantitativa, desenvolvido junto a 80 alunos matriculados no primeiro e oitavo semestre dos cursos de medicina e enfermagem de um centro universitário do Acre, durante o segundo semestre do calendário acadêmico de 2019. A seleção dos estudantes ocorreu através de amostra não probabilística ou por conveniência, onde os acadêmicos que atendiam os critérios de inclusão do estudo foram convidados a participar do estudo. Cada turma dos cursos selecionados para a amostra, contava em média com 40 alunos, sendo assim, foram definidos que 20 de cada turma participaria do estudo.

O centro universitário onde o estudo foi realizado fica localizado no estado no Acre, na capital Rio Branco, e oferece atualmente mais de 20 cursos nas mais diversas áreas do conhecimento. O bloco da saúde que comporta os cursos de medicina e enfermagem possui $16.000 \mathrm{M}^{2}$, com amplas salas e laboratórios modernos, dentre esses, um centro de simulação realística, um dos mais modernos do país, voltado para cursos das áreas de saúde.

Os critérios de inclusão definidos para o estudo foram: o estudante estar matriculado no primeiro e oitavo semestres dos cursos de medicina e enfermagem, no segundo semestre do calendário acadêmico de 2019 e aceitarem participar do estudo. Foram excluídos os estudantes que não compareceram à instituição na data da coleta de dados.

Os procedimentos metodológicos de coleta de dados incluíram: após a aprovação da pesquisa no comitê de ética local, os pesquisadores se dirigiram as salas de aula em horário previamente agendado com as coordenações dos cursos e professores. Após a exposição dos objetivos da pesquisa, os primeiros 20 alunos que manifestaram o desejo de participar permaneceram na sala de aula, os demais foram liberados. Após esse momento, foi procedida a leitura e assinatura do termo de consentimento livre e esclarecido e a coleta de dados ocorreu de maneira individual, na sala de aula dos estudantes, onde foi preenchido pelos próprios estudantes, um instrumento de auto avaliação denominado Escala de Ansiedade, Depressão e Estresse-21 (EADS - 21), bem como uma ficha contendo os dados sócio demográficos dos estudantes.
A EADS - 21 é uma escala de auto relato, com três subescalas (depressão, ansiedade e estresse), tipo Likert, constituída por 7 itens. Cada item consiste numa frase (uma afirmação) que remete para sintomas emocionais negativos. Pede-se aos sujeitos que respondam em que medida cada afirmação se the aplicou durante o último mês. Para cada frase existem quatro possibilidades de resposta, apresentadas numa escala tipo Likert de 4 pontos (de 0 a 3: 0. "não se aplicou nada a mim", 1. "aplicou-se a mim algumas vezes", 2. "aplicou-se a mim muitas vezes" 3. "aplicou-se a mim maior parte das vezes"). A pontuação de cada subescala ou fator corresponde à soma das pontuações dos respetivos itens (Furtado, 2016).

Para obtenção dos resultados, foi utilizada a versão portuguesa da escala EADS-21, sendo considerados elevados os índices ( 0,85 para a depressão, 0,74 para a ansiedade e de 0,81 para o estresse) (Pais Ribeiro et al., 2004).

A EADS - 21 não dá diagnóstico de depressão, sendo utilizado apenas para rastrear sintomas depressivos de ansiedade e estresse na população. Para o estabelecimento do diagnóstico, após a obtenção do resultado, o indivíduo precisa ser avaliado por um especialista médico.

Após essa fase, realizaram-se análises descritivas a partir da média e da frequência dos dados coletados. Os dados foram analisados criteriosamente, e as análises foram conduzidas usando-se a frequência absoluta e percentual. Para produção dos gráficos e tabelas, foi utilizada a ferramenta do Microsoft Office Excel 2010.

A pesquisa foi autorizada pelo comitê de ética em pesquisa com seres humanos local sob CAAE 10999019.8.0000.8028.

\section{Resultados}

A amostra obtida constituiu-se de 80 alunos com idade média de 22,05 anos. Contudo, é possível analisar com especificidade todas as análises feitas a partir dos dados coletados deste total de estudantes, os quais foram colocados em tabelas para melhor compreensão dos leitores. 
Tabela 1. Dados sócio demográficos dos estudantes de um centro universitário do Acre em 2019 (n=80)

\begin{tabular}{lr}
\hline VARIÁVEL & ANÁLISE DESCRITIVA \\
\hline Idade & $\mathbf{n}(\%)$ \\
15 a 19 anos & $21(26,0 \%)$ \\
20 a 29 anos & $46(57,0 \%)$ \\
30 a 39 anos & $6(8,0 \%)$ \\
40 a 40 anos & $3(4,0 \%)$ \\
Não respondeu & $3(5,0 \%)$ \\
Sexo & \\
Feminino & $52(65 \%)$ \\
Masculino & $28(35 \%)$ \\
Estado Civil & \\
Solteiro & $63(79 \%)$ \\
Casado & $12(15 \%)$ \\
Outros & $5(6 \%)$ \\
Local de Procedência & \\
Dentro do Estado & $61(76 \%)$ \\
Fora do Estado & $19(24 \%)$ \\
Situação de Moradia & $19(24 \%)$ \\
Mora sozinho & $61(76 \%)$ \\
Mora com outros & \\
Atividade remunerada & $65(81 \%)$ \\
Nenhuma & $15(19 \%)$ \\
Trabalho remunerado & \\
\hline
\end{tabular}

Concernente aos dados expostos na tabela 1, observa-se que, quanto ao sexo, a maioria dos participantes eram do sexo feminino 52 (65\%). Quanto ao estado civil, a maior parte 63 (79\%) eram solteiros. Ao total, 61 (76\%) eram procedentes do estado do Acre. Em termos de moradia, a maioria 61(76\%) afirmou residir com outras pessoas. Quanto à atividade remunerada 65 (81\%) alegaram ainda não exercer.

Tabela 2. Aspectos da saúde mental dos estudantes de um centro universitário do Acre em 2019 ( $n=80)$

\begin{tabular}{|c|c|}
\hline VARIÁVEL & ANÁLISE DESCRITIVA \\
\hline \multicolumn{2}{|c|}{ Já fez psicoterapia? } \\
\hline Sim & $23(29 \%)$ \\
\hline Não & 48 (60\%) \\
\hline Em andamento & $7(9 \%)$ \\
\hline Não respondeu & $2(2 \%)$ \\
\hline \multicolumn{2}{|c|}{ Já fez tratamento psiquiátrico? } \\
\hline Sim & $8(10 \%)$ \\
\hline Não & $57(71 \%)$ \\
\hline Não respondeu & $12(15 \%)$ \\
\hline Em andamento & $3(4 \%)$ \\
\hline \multicolumn{2}{|c|}{ Faz atividades de lazer? } \\
\hline Sempre & $26(33 \%)$ \\
\hline Esporadicamente & $53(66 \%)$ \\
\hline Não respondeu & $1(1 \%)$ \\
\hline
\end{tabular}

Mediante aos dados demostrados na tabela 2, a maior parte dos participantes 48 (60\%) afirmou nunca ter realizado psicoterapia. A respeito da realização de tratamento psiquiátrico anterior, 57 (71\%) afirmou nunca ter realizado, sendo que referente a realização de atividades de lazer, a maioria 53 (66\%) evidenciou que praticava esporadicamente. 
Gráfico 1. Uso de medicamentos para o desempenho acadêmico entre estudantes dos cursos de enfermagem e medicina de um Centro Universitário do Acre em $2019(n=80)$

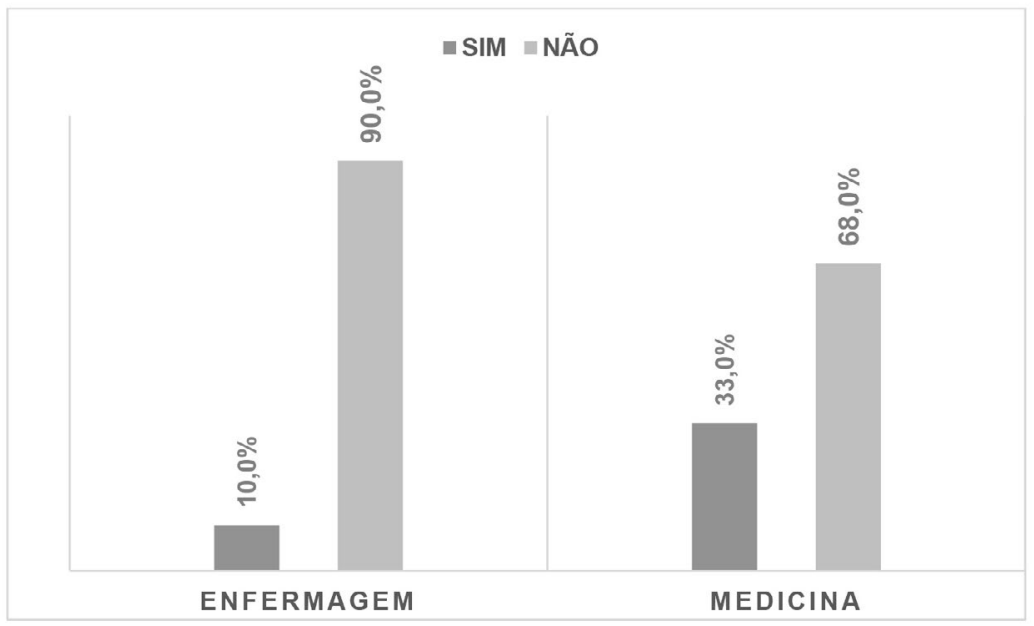

Em relação ao hábito de fazer uso de medicamentos para aumento de desempenho acadêmico (gráfico 1), observou-se que entre os estudantes de enfermagem 36 (90\%) nunca haviam feito uso, e dentre os acadêmicos de medicina a maioria também 27 (68\%), não haviam feito o uso.

Tabela 3. Média de Sintomas de Depressão entre estudantes dos cursos de enfermagem e medicina de um Centro Universitário do Acre em 2019 ( $n=80$ )

\begin{tabular}{ccccccccc}
\hline CURSOS & \multicolumn{7}{c}{ ITENS DA ESCALA EADS - 21 } & \multicolumn{1}{c}{ Média } \\
\cline { 2 - 10 } & $\mathbf{3}$ & $\mathbf{5}$ & $\mathbf{1 0}$ & $\mathbf{1 3}$ & $\mathbf{1 6}$ & $\mathbf{1 7}$ & $\mathbf{2 1}$ & $\mathbf{1}$ \\
\hline Enfermagem & 1,58 & 1,61 & 1,53 & 1,48 & 1,37 & 1,36 & 1,39 & $\mathbf{1 , 4 7}$ \\
\hline Medicina & 0,95 & 0,95 & 0,91 & 0,92 & 0,78 & 0,80 & 0,77 & $\mathbf{0 , 9}$
\end{tabular}

Concernente aos dados expostos na tabela 3 quanto as médias de sintomas de depressão entre estudantes de medicina e enfermagem, observa que a média dos estudantes de enfermagem apresenta-se maior $(1,47)$ do que dos acadêmicos de medicina $(0,9)$.

Tabela 4. Média de Sintomas de Ansiedade entre estudantes dos cursos de enfermagem e medicina de um Centro Universitário do Acre em 2019 ( $\mathrm{n}=80$ )

\begin{tabular}{|c|c|c|c|c|c|c|c|c|}
\hline \multirow[t]{2}{*}{ CURSOS } & \multicolumn{7}{|c|}{ ITENS DA ESCALA EADS - 21} & \multirow[b]{2}{*}{ Média } \\
\hline & 2 & 4 & 7 & 9 & 15 & 19 & 20 & \\
\hline Enfermagem & 1,10 & 1,13 & 1,17 & 1,13 & 1,06 & 1,09 & 1,12 & 1,11 \\
\hline Medicina & 0,85 & 0,88 & 0,93 & 0,93 & 0,86 & 0,85 & 0,79 & 0,87 \\
\hline
\end{tabular}

A Tabela 4 evidencia a média de sintomas de ansiedade entre estudantes dos cursos de medicina e enfermagem e observa-se que os estudantes de enfermagem apresentaram maior média $(1,11)$, quando comparados aos do curso de medicina $(0,87)$. 
Tabela 5. Média de Sintomas de Estreses entre estudantes dos cursos de enfermagem e medicina de um Centro Universitário do Acre em 2019 ( $\mathrm{n}=80$ )

\begin{tabular}{ccccccccc}
\hline CURSOS & \multicolumn{7}{c}{ ITENS DA ESCALA EADS - 21 } & \\
\cline { 2 - 8 } & $\mathbf{1}$ & $\mathbf{6}$ & $\mathbf{8}$ & $\mathbf{1 1}$ & $\mathbf{1 2}$ & $\mathbf{1 4}$ & $\mathbf{1 8}$ & Média \\
\hline Enfermagem & 1,25 & 0,87 & 1,90 & 1,55 & 1,48 & 1,08 & 1,95 & $\mathbf{1 , 4 4}$ \\
\hline Medicina & 1,48 & 1,40 & 1,61 & 1,61 & 1,51 & 1,12 & 1,49 & $\mathbf{1 , 4 6}$ \\
\hline
\end{tabular}

Os dados evidenciados na Tabela 5 demostram que houve maior tendência aos sintomas de estresse entre acadêmicos de medicina $(1,46)$ em relação aos alunos de enfermagem, apresentando $(1,44)$.

Gráfico 2. Média de sintomas de Depressão, Ansiedade e Estresse por período entre estudantes dos cursos de enfermagem e medicina de um Centro
Universitário do Acre em $2019(n=80)$

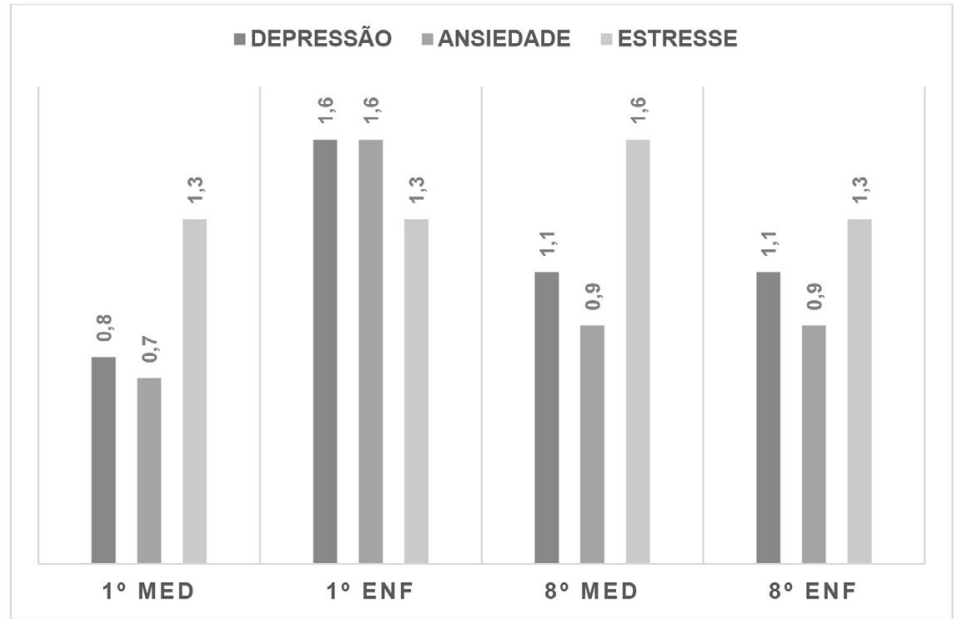

Concernente aos dados evidenciados no Gráfico 2, referente à média de sintomas de depressão, ansiedade e estresse por período entre estudantes dos cursos de enfermagem e medicina em comparativo entre os primeiros períodos dos cursos, o primeiro período de enfermagem apresentou maiores sintomas de depressão, ansiedade e estresse, tendo como média $(1,6) ;(1,6)$, respectivamente, sendo que, em relação ao estresse, foram encontrados os mesmos índices para ambos os cursos $(1,3)$.

Levando em consideração os sintomas dos acadêmicos do $8^{\circ}$ período de ambos os cursos, verifica-se que os alunos do $8^{\circ}$ período de medicina e enfermagem obtiveram as mesmas médias, com $(1,1)$ para depressão $(0,9)$ para ansiedade, no entanto quanto aos sintomas de estresse, os alunos de medicina obtiveram a média de $(1,6)$, enquanto que os de enfermagem esse índice foi de $(1,3)$.

\section{Discussão}

De forma geral, o presente estudo pretendeu evidenciar os índices de depressão ansiedade e estresse entre estudantes de um centro universitário do Acre. Nesse sentido, os achados de Leão et al (2018), que desenvolveu um estudo semelhante, referente a prevalência e fatores associados à depressão e ansiedade entre estudantes universitários da área da saúde de um grande centro urbano do Nordeste do Brasil, evidenciou o predomínio de estudantes do sexo feminino com $71,6 \%$ sendo que a maioria $92,0 \%$ eram solteiros, $80,7 \%$ não exerciam nenhuma atividade remunerada e $77,9 \%$ residiam com os pais, corroborando com os nossos achados. 
Os resultados encontrados por Ariño e Bardagi (2018) também vão de encontro com os achados do presente estudo, uma vez que a média de idade dos participantes foi de 23,7 anos, sendo que a maioria dos estudantes era mulheres, solteiras, não possuíam filhos, não exerciam atividade remunerada e residiam com outras pessoas. Também, o estudo de Fernandes et al. (2018) aponta para uma preponderância do gênero feminino no ambiente universitário $(81,5 \%)$, solteiros (as) $(91,6 \%)$, natural da capital do estado em estudo $(70,2 \%)$, e que ainda moravam com os pais $(73,0 \%)$.

No que concerne à questão da maioria dos estudantes residirem com a família, a literatura afirma que esse é um fator positivo para evitar o desenvolvimento de crises emocionais, uma vez que os achados de Vasconcelos et al. (2015) constataram que há maior risco de depressão entre os estudantes universitários procedentes de municípios distantes da universidade e que, consequentemente, estavam afastados do âmbito familiar, tornando-se mais expostos a distúrbios psicológicos. Por outro lado, ele afirma que o fato de o estudante dispor de pessoas próximas, com quem possa compartilhar sentimentos, é um elemento importante para a sua saúde mental.

Em referência ao fato de a maioria dos estudantes não desenvolverem atividades remuneradas, o estudo de Rosa e Ribeiro (2017) afirma que os aspectos da vida financeira de um estudante de ensino superior podem afetar seu desenvolvimento acadêmico e sua saúde mental de diversas formas, uma vez que os desafios socioeconômicos são um dos principais fatores responsáveis pela evasão do sistema de educação superior, bem como serem responsáveis pelo desenvolvimento de transtornos mentais.

Quanto ao fato de que a maioria dos estudantes não realizou psicoterapia anterior e nem atendimento psiquiátrico, esses achados divergem do resultado do relatório do perfil socioeconômico e cultural dos estudantes de graduação das universidades federais brasileiras (Fornaprace, 2011), conforme citado anteriormente, onde a maioria dos estudantes que tinha queixas de sofrimento psíquico procuraram atendimento psicológico e também haviam procurado atendimento psiquiátrico.
O estudo realizado por Oliveira (2013) junto a estudantes de Medicina da Universidade Federal da Bahia evidenciou que em relação à realização de tratamento psicoterápico, $17,2 \%$ dos estudantes afirmaram que já haviam realizado e 3,5\% estavam em tratamento. Sendo que $7 \%$ dos participantes já haviam se submetido a tratamento psiquiátrico. No tocante a realização de atividades de lazer, 39,7\% relataram realizar atividades de lazer apenas esporadicamente.

Referindo-se ao estudo desenvolvido por Abrão et al. (2008), os autores evidenciam que, quanto às atividades de lazer, observou-se que $43,50 \%$ dos acadêmicos sempre as praticavam, divergindo dos nossos achados. Os resultados de uma pesquisa realizada com universitários do primeiro ano da Southern Illinois University School of Medicine concluiu que os alunos com elevados escores de estresse e ansiedade eram aqueles que exerciam nenhuma ou pouca atividade de lazer (Folse et al., 1985).

Destarte, os resultados do estudo de Botti (2010) em estudantes do curso de enfermagem evidenciou que houve uma prevalência de $19,08 \%$ no uso de ansiolíticos/ medicamentos para melhorar no desempenho acadêmico. Outro estudo realizado na Universidade Federal de Tocantins por Moraes et al. (2019) revelou que estimulantes sem receita médica apresentou um percentual de ocorrência de $27 \%$ em homens e 14,9\% em mulheres no curso de medicina, sendo a Ritalina e o pó de guaraná as substancias mais utilizadas pelos estudantes.

Cabe salientar que, segundo o estudo de Leão et al. (2018), a prevalência de pessoas com o uso de substâncias estimulantes foi de $57,5 \%$, sendo que $51,3 \%$ desses começaram a fazer o uso durante a faculdade, dentre essas, destaca-se o consumo de metilfenidato (Ritalina $\AA$ ), onde $64 \%$ iniciaram o consumo durante o curso de Medicina, conforme citado anteriormente no estudo de Pires et al. (2018).

Os resultados encontrados no presente estudo, no que se refere ao aparecimento de depressão entre os estudantes, corroboram com recente pesquisa realizada com estudantes da área de saúde e desenvolvida por Leão et al. (2018), como citada na introdução do presente estudo, onde observa-se um crescente aumento de casos de sintomas de sofrimento psíquico entre eles. 
De igual forma, os achados do estudo de Camargo et al. (2014), realizado com estudantes do curso de enfermagem, também identificou sintomas de depressão na maioria dos indivíduos pesquisados (62,6\%). Resultado semelhante também encontrado no estudo de Deperon et al. (2013), onde foi possível identificar sintomas de depressão entre alunos do curso de enfermagem, identificando ainda que eles estão suscetíveis a experiências estressantes, como a exigência de um bom desempenho acadêmico, dentre essas aulas práticas e a realização de plantões obrigatórios nos estágios supervisionados, o que pode contribuir para o aparecimento dos sintomas.

No que se refere aos resultados encontrados entre estudantes do curso de medicina, nossos achados estão em consonância com o estudo realizado por Amaral et al. (2008), onde a maioria dos estudantes, $73,2 \%$, não apresentou sintomas considerados depressivos, bem como com o estudo de Lima et al. (2010), onde estudantes de medicina, 52\%, não apresentaram sintomas depressivos. No entanto, de forma divergente, nos achados de Costa et al. (2020), dos estudantes do curso de medicina participantes do estudo, $28 \%$ apresentavam sintomas de depressão, sendo $51,3 \%$ com sinais de depressão de leve a moderada, 35,9\% com sinais de depressão moderada e $12,8 \%$ com sinais de depressão severa.

Ainda de acordo com o relatório do perfil socioeconômico e cultural dos estudantes de graduação das universidades federais brasileiras Fonaprace (2011), cerca de $60 \%$ dos casos de sintomas depressivos podem ser precedidos pela ocorrência de fatores estressantes, principalmente de origem psicossocial, dentre esses, o stress e o esgotamento físico, uma vez que o ambiente universitário com a carga pesada de trabalhos acadêmicos e fatores não diretamente ligados à vida acadêmica (como moradia, condições financeiras e habilidades sociais), porém relacionados com as dificuldades acadêmicas, podem levar ao desenvolvimento da doença.

Os resultados do estudo de Ibrahim (2013) apontam que os índices de ansiedade e depressão encontrados nos estudantes universitários são elevados, sendo, inclusive, muito superiores aos encontrados na população geral, com os alunos dos primeiros anos da universidade apresentando a maior concentração de sintomas depressivos e de ansiedade.
Frente a isso, os achados de Marchi et al. (2013), em um estudo realizado com estudantes de ensino superior da área da saúde, evidenciaram que os estudantes dessa área apresentam maior nível de ansiedade, quando comparados a outras áreas de ensino. Ele ainda afirma que isso pode estar relacionado à experiência da prática clínica, como o lidar com o ser humano, o contato com o sofrimento psíquico, a observação constante dos instrutores no cenário da prática, o medo de cometer erros e sentimentos de inadequação ao ambiente hospitalar que é dinâmico e intenso.

Um estudo desenvolvido por Ferreira (2014) sobre a ansiedade antes da realização de avaliações em estudantes universitários encontrou níveis altos e médios de ansiedade entre eles, justificados pelos desafios intelectuais enfrentados, gerando tensões e culminando em episódios de ansiedade.

Nesse sentido, alerta-se para a gravidade do problema, uma vez que a ansiedade pode afetar diversas áreas da vida dos universitários e atingir grandes proporções na vida pessoal e diretamente na vida acadêmica, o que traz uma preocupação no uso indevido de medicamentos, conforme evidenciado por Pires et al. (2018), além de outros agravos na vida dos mesmos. Mediante a isso, diferentes estratégias devem ser utilizadas para o enfrentamento desse quadro, como redes de apoio para os estudantes dentro das universidades, como por exemplo o incentivo para envolvimento deles com atividades recreativas, a disponibilização de aconselhamento individual, através de sessões de psicoterapia ou ainda a implementação de atividades baseadas na religião ou espiritualidade da pessoa (Costa, 2017; Chaves, 2015).

Frente aos achados do presente estudo, que evidenciaram maior índice de estresse entre os acadêmicos de medicina, esse resultado vai ao encontro das prevalências encontradas na pesquisa de Aguiar et al. (2008), na qual houve uma maior ocorrência de sintomas de estresse de 49,7\% ( $n=99$ ) entre eles, observando-se que não houve diferença entre as fases do estresse e os sintomas predominantes entre os semestres avaliados.

Os achados de Botti et al. (2010) também destacam a prevalência dos sintomas de estresse em estudantes de medicina, sendo que os fatores 
geradores do estresse nos mesmos foram: a transição em relação ao estilo de vida; grande demanda de informações a aprender; dificuldade em conciliar o estudo com as atividades de lazer e tarefas domésticas; competição entre os estudantes e problemas pessoais e financeiros.

Os estudantes, muito antes de adentrarem a universidade, passam por diversas exigências, como cursos preparatórios, vestibulares, cobranças dos pais, resultando no ingresso na universidade já manifestando sintomas de estresse, sendo assim, em conformidade com a pesquisa realizada em estudantes de enfermagem por Cestari et al. (2017), os resultados apontaram para a ocorrência de $(26,1 \%)$ de estresse entre os acadêmicos do primeiro ano, tendo aumento gradativo com o decorrer da graduação.

Dessa forma, os achados do estudo de Bayram e Bilgel (2008) evidenciam que a população universitária está vulnerável ao desenvolvimento de transtornos mentais, como a depressão, a ansiedade e o estresse.

Corroborando com os achados da presente pesquisa, onde os estudantes de enfermagem apresentaram mais sintomas de depressão e ansiedade do que os estudantes do curso de medicina, os resultados do estudo realizado por Leão et al. (2018) com estudantes universitários da área da saúde no Nordeste do Brasil demostrou que os estudantes do curso de medicina, embora sejam muito mais cobrados em quesitos de carga horária e desempenho, não obtiveram as maiores porcentagens em relação aos sintomas de depressão e ansiedade, frente aos estudantes dos outros cursos.

De acordo com a pesquisa realizada por Fernandes (2018), os estudantes universitários apresentam sintomas depressivos acima do encontrado na população não universitária, o que pode ser consequência do estilo de vida, da competitividade e às cobranças por bons desempenhos, entre outros fatores.

Conforme evidenciado, quanto ao índice de estresse, os acadêmicos de ambos os cursos, apresentaram resultados semelhantes, principalmente no início da vida acadêmica, dessa forma, os ingressantes podem estar expostos a níveis de estresse nessa fase, uma vez que ao entrarem para o ambiente universitário podem vivenciar situações de estresse para se adaptar ao novo ambiente e à nova condição, com novas obrigações escolares, responsabilidades do processo de formação universitária, necessidade de organização de tarefas, adaptação do aluno a critérios e formas de avaliação, entre outras (Bublitz et al, 2016), no entanto, assim eles também podem apresentar sintomas de depressão e ansiedade nessa fase, devido à pressão por bom desempenho acadêmico, e o medo do novo, deixando-os mais vulneráveis psicologicamente.

De acordo com os achados de Moreira \& Furegato (2013), os estudantes dos últimos semestres também podem estar expostos a sintomas de estresse, por estarem mais preocupados com a futura inserção no mercado de trabalho, inseridos nos campos de prática, expostos ao enfretamento de conflitos com outros profissionais e à competitividade com os colegas de turma.

Sendo assim, estudos realizados ao longo dos anos com estudantes universitários, principalmente das áreas de saúde, vêm apontando que o estresse pode gerar consequências negativas a eles, como o surgimento de transtornos de ansiedade e de humor, prejudicando o seu desempenho acadêmico, diminuindo a atenção e concentração, podendo afetar as habilidades de tomar decisões, interferindo no estabelecimento de uma relação efetiva com o paciente. Mediante a isso, o estresse nessa população é motivo de preocupação, pois a longo prazo pode desencadear um problema de saúde pública, uma vez que, ao prejudicar a educação dos futuros profissionais, acarretará malefícios que poderão recair também sobre os usuários (Padovani et al., 2014; Vasconcelos et al., 2015; Lima et al., 2016).

Nesse sentido, esses fatores podem prejudicar a saúde mental dos estudantes, necessitando, dessa forma, que as instituições que os acolhe promovam atividade de apoio, cuidado e orientação, afim de evitar o transtorno mental. (Bolsoni-Silva; Guerra, 2014).

Como limitações do presente estudo, destaca-se que se tratando de uma amostra não probabilística (por conveniência) reveste-se das limitações inerentes a ela, na qual os resultados podem não ser representativos da totalidade dos estudantes. 


\section{Considerações finais}

Ao final do estudo, conclui-se que a saúde mental dos estudantes pode estar em risco. Dessa forma, os resultados encontrados tornam-se válidos, pois alcançaram os objetivos propostos, fornecendo dados relevantes, para se pensar a saúde mental dos mesmos, o que evidencia a necessidade da implementação de um projeto político-pedagógico institucional que vise o bem-estar dessa população e a promoção da saúde mental, diagnóstico e tratamento precoce.

Reafirma-se que as particularidades inerentes às exigências do ensino superior têm impacto significativo na qualidade de vida e na saúde mental dos acadêmicos e que as políticas públicas de acesso e permanência nas universidades devem considerar tal problemática no desenvolvimento de suas ações.

Sugere-se, assim, novas pesquisas que busquem ampliar o conhecimento acerca das variáveis acadêmicas. Também se sugere a construção de instrumentos que permitam avaliar a saúde mental da população universitária, considerando as especificidades da população em questão.

\section{Contribuições dos autores}

Santiago MB, Braga OS, Silva PR e Capelli VMR participaram da concepção, delineamento, coleta, interpretação e análise dos dados, e redação do artigo científico. Costa RSL participou da concepção, delineamento, interpretação e análise dos dados, e redação do artigo científico. Supervisionou e orientou o estudo.

\section{Conflitos de interesses}

Nenhum conflito financeiro, legal ou político envolvendo terceiros (governo, empresas e fundações privadas, etc.) foi declarado para nenhum aspecto do trabalho submetido (incluindo, mas não se limitando a subvenções e financiamentos, participação em conselho consultivo, desenho de estudo, preparação de manuscrito, análise estatística, etc.).

\section{Referências}

Abrão, C. B., Coelho, E. P., \& Passos, L. B. S. (2008). Prevalência de sintomas depressivos entre estudantes de medicina da Universidade Federal de Uberlândia. de medicina da Universidade Federal de Uberlândia. Revista brasileira de educação médica, 32(3), 315-323. https://doi.org/10.1590/ S0100-55022008000300006

Aguiar, S. M., Vieira, A. P. G. F., Vieira, K. M. F., Aguiar, S. M., \& Nóbrega, J. O. (2009). Prevalência de sintomas de estresse nos estudantes de medicina. Jornal Brasileiro De Psiquiatria, 58(1), 34-38. https://doi.org/10.1590/5004720852009000100005

Amaral, G. F., Gomide, L. M. P., Batista, M. P., Píccolo, P. P., Teles, T. B. G., Oliveira, P. M., \& Pereira, M. A. D. (2008). Sintomas depressivos em acadêmicos de medicina da Universidade Federal de Goiás: um estudo de prevalência. Revista de Psiquiatria do Rio Grande do Sul, 30(2), 124-130. https://doi. org/10.1590/S0101-81082008000300008

Ariño, D. O., \& Bardagi, M. P. (2018). Relação entre Fatores Acadêmicos e a Saúde Mental de Estudantes Universitários. Revista Psicologia em Pesquisa, 12(3), 44-52. https://dx.doi.org/10.24879/2018001200300544

Fernandes, M. A., Vieira, F. E. R., Silva, J. S., Avelino, F. V. S. D., \& Santos, J. D. M. (2018). Prevalência de sintomas ansiosos e depressivos em universitários de uma instituição pública. Revista Brasileira de Enfermagem, 71(Supl. 5), 2169-2175. https://doi.org/10.1590/0034-7167-2017-0752

Alves, T.C.T.F. (2014). Depressão e ansiedade entre estudantes da área de saúde. Revista de Medicina, 93(3), 101-105. https:// doi.org/10.11606/issn.1679-9836.v93i3p101-105

Bayram, N., \& Bilgel, N. (2008). The prevalence and sociodemographic correlations of depression, anxiety and stress among a group of university students [A prevalência e as correlações sociodemográficas de depressão, ansiedade e estresse entre um grupo de estudantes universitários]. Social Psychiatry And Psychiatric Epidemiology, 43(8), 667-672. https://doi.org/10.1007/ s00127-008-0345-x

Bolsoni-Silva, A.T., \& Guerra, B.T. (2014). O impacto da depressão para as interações sociais de universitários. Estudos $e$ Pesquisas em Psicologia, 14(2), 429-452. https://repositorio. unesp.br/handle/11449/135634

Botti, N. C., Lima, A. F. D., \& Simões, W. M. B. (2010). Uso de substâncias psicoativas entre acadêmicos de enfermagem da Universidade Católica de Minas Gerais. Revista Eletrônica Saúde Mental Álcool E Drogas, 6(1), 1-20. http://pepsic.bvsalud.org/scielo.php?script=sci_ arttext\&pid=S1806-69762010000100013 
Bublitz, S., Guido, L. A., Lopes, L. F. D., \& Freitas, E. O. (2016). Associação entre estresse e características sociodemográficas e acadêmicas de estudantes de enfermagem. Texto \& Contexto-Enfermagem, 25(4), e2440015. https://doi.org/10.1590/010407072016002440015

Camargo, R. M., Sousa, C. O., \& Oliveira, M. L. C. (2014). Prevalência de casos de depressão em acadêmicos de enfermagem em uma instituição de ensino de Brasília. Revista Mineira de Enfermagem, 18(2), 392-403. http://www. dx.doi.org/10.5935/1415-2762.20140030

Cestari, V. R. F., Barbosa, I. V., Florêncio, R. S., Pessoa, V. L. M. P., \& Moreira, T. M. M. (2017). Estresse em estudantes de enfermagem: estudo sobre vulnerabilidades sociodemográficas e acadêmicas. Acta Paulista de Enfermagem, 30(2), 190-196. https://doi.org/10.1590/19820194201700029

Costa, K. M. V., Sousa, K. R. S., Formiga, P. A., Silva, W. S., \& Bezerra, E. B. N. (2017, June). Ansiedade em universitários na área da saúde. In // Congresso Brasileiro das Ciências da Saúde. https://www.editorarealize.com.br/editora/ anais/conbracis/2017/TRABALHO EV071 MD1 SA13 ID592_14052017235618.pdf

Costa, D. S., Medeiros, N. S. B., Cordeiro, R. A., Frutuoso, E. S., Lopes, J. M., \& Moreira, S. N. T. (2020). Sintomas de Depressão, Ansiedade e Estresse em Estudantes de Medicina e Estratégias Institucionais de Enfrentamento. Revista Brasileira de Educação Médica, 44(1), e040. https:// dx.doi.org/10.1590/1981-5271v44.1-20190069

Chaves, E. C. L., lunes, D. H., Moura, C. C., Carvalho, L. C., Silva, A. M., \& Carvalho, E. C. (2015). Ansiedade e espiritualidade em estudantes universitários: um estudo transversal. Revista brasileira de enfermagem, 68(3), 504-509. http:// dx.doi.org/10.1590/0034-7167.2015680318i

Claudino, J., \& Cordeiro, R. (2006). Níveis de ansiedade e depressão nos alunos do curso de licenciatura em enfermagem. O caso particular dos alunos da Escola Superior de Saúde de Portalegre. Millenium, 32(11), $197-$ 210. https://revistas.rcaap.pt/millenium/article/view/8403

Deperon, C. O., Pereira C. B., Teixeira C.P.S., Pereira F.G.F., \& Silva, M.G. (2013) Comportamento depressivo em acadêmicos de enfermagem. In Seminário Nacional de Pesquisa em Enfermagem. http://www.abeneventos.com.br/anais senpe/17senpe/pdf/0669po.pdf

Ferreira, E. S. D. (2014). Ansiedade aos exames em estudantes universitários: relação com stresse académico, estratégias de coping e satisfação académica [Dissertação de mestrado, Universidade Lusófona de Humanidades e Tecnologia]. https://recil.grupolusofona.pt/bitstream/10437/6021/1/ Elisabete\%20Ferreira\%20tese\%20final.pdf
Folse, M. L., DaRosa, D. A., \& Folse, R. (1985). The relationship between stress and attitudes toward leisure among firstyear medical students [A relação entre estresse e atitudes em relação ao lazer entre estudantes de medicina do primeiro ano]. Journal of Medical Education, 60(8), 610-617. https://doi.org/10.1097/00001888-198508000-00004

FONAPRACE (2011). Perfil Socioeconômico e Cultural dos Estudantes de Graduação das Universidades Federais Brasileiras. ANDIFES. RepositóriUM. http://flacso.redelivre.org.br/ files/2012/08/807.pdf

Furtado, C. F. (2016). Implicações do stresse no presentismo das costureiras da industria têxtil em Portugal [Tese de doutorado, Universidade do Minho]. http://hdl.handle. net/1822/48008

Furtado, E. S., Falcone, E. M. O., \& Clark, C. (2003). Avaliação do estresse e das habilidades sociais na experiência acadêmica de estudantes de medicina de uma universidade do Rio de Janeiro. Interação em Psicologia, 7(2), 43-51. http://dx.doi.org/10.5380/psi.v7i2.3222

Geraldo, J.B. (2020). Estresse, Ansiedade e Esgotamento. Cérebro e Mente: Revista Eletrônica de Divulgação Científica em Neurociência. https://cerebromente.org.br/n11/doencas/ estresse.htm

Ibrahim, A. K., Kelly, S. J., Adams, C. E., \& Glazebrook, C. (2013). A systematic review of studies of depression prevalence in university students [Uma revisão sistemática de estudos de prevalência de depressão em estudantes universitários]. Journal of Psychiatric Research, 47(3), 391400. https://doi.org/10.1016/j.jpsychires.2012.11.015

Jardim, S. (2011). Depressão e trabalho: ruptura de laço social. Revista Brasileira de Saúde Ocupacional, 36(123), 84-92. https://doi.org/10.1590/S0303-76572011000100008

Leão, A. M., Gomes, I. P., Ferreira, M. J. M., \& Cavalcanti, L. P. G. (2018). Prevalência e fatores associados à depressão e ansiedade entre estudantes universitários da área da saúde de um grande centro urbano do Nordeste do Brasil. Revista brasileira de educação médica, 42(4), 55-65. https:// doi.org/10.1590/1981-52712015v42n4rb20180092

Lima, L. S., Ferry, V., Fonseca, R. N. M., Silva Junior, G. F., \& Jadão, F. R. S. (2010). Sintomas depressivos nos estudantes de medicina da Universidade Estadual do Maranhão. Revista Neurociências, 18(1), 8-12. http:// www.revistaneurociencias.com.br/edicoes/2010/ RN1801/366\%20original.pdf

Lima, R. L., Soares, M. E. C., Prado, S. N., \& Albuquerque, G. S. C. (2016). Estresse do Estudante de medicina e rendimento acadêmico. Revista Brasileira de Educação Médica, 40(4), 678-684. https://doi.org/10.1590/1981$\underline{52712015 \mathrm{v} 40 \mathrm{n} 4 \mathrm{e} 01532015}$ 
Marchi, K. C., Bárbaro, A. M., Miasso, A. I., \& Tirapelli, C. R. (2013). Ansiedade e consumo de ansiolíticos entre estudantes de enfermagem de uma universidade pública. Revista Eletrônica de Enfermagem, 15(3), 729-37. https://doi. org/10.5216/ree.v15i3.18924

Moraes, D. P. A., Medeiros, G. M. R., Caldas, F. A. X. B., Oliveira, L. A., \& Baldaçara, L. (2018). Prevalência do uso de drogas psicotrópicas por estudantes de medicina da Universidade Federal do Tocantins. Arquivos Médicos dos Hospitais e da Faculdade de Ciências Médicas da Santa Casa de São Paulo, 58(3), 127-133. http://arquivosmedicos.fcmsantacasasp. edu.br/index.php/AMSCSP/article/view/237

Moreira, D. P., \& Furegato, A. R. F. (2013). Estresse e depressão entre alunos do último período de dois cursos de enfermagem. Revista latino-americana de enfermagem, 21(spe), 155-162. https://doi.org/10.1590/S010411692013000700020

Oliveira, E. N. (2013). Prevalência de sintomas depressivos em estudantes de Medicina da Universidade Federal da Bahia [Monografia, Universidade Federal da Bahia]. Repositório Institucional UFBA. https://repositorio.ufba.br/ri/ bitstream/ri/13959/1/Elis\%C3\%A2ngela\%20Neves\%20 de\%200liveira.pdf

Padovani, R. C., Neufeld, C. B., Maltoni, J., Barbosa, L. N. F., Souza, W. F., Cavalcanti, H. A. F., \& Lameu, J. N. (2014). Vulnerabilidade e bem-estar psicológicos do estudante universitário. Revista brasileira de terapias cognitivas, 10(1), 02-10. https://dx.doi.org/10.5935/1808-5687.20140002
Pais-Ribeiro, J. L, Honrado, A., \& Leal, I. (2004). Contribuição para o Estudo da Adaptação Portuguesa das Escalas de Ansiedade, Depressão e Stress (EADS) de 21 Itens de Lovibond e Lovibond. Psicologia, Saúde e Doenças, 5(2), 229-239. http://www.scielo.mec.pt/pdf/psd/v5n2/v5n2a07. pdf

Pires, M. S., Dias, A. P., Pinto, D. C. L., Gonçalves, P. G., \& Segheto, W. (2018). O uso de substâncias psicoestimulantes sem prescrição médica por estudantes universitários. Revista Cientifica FAGOC-Saúde, 3(2), 22-29. https://revista.fagoc.br/ index.php/saude/article/view/370

Rosa, C. M., \& Ribeiro, R. (2017). Percalços da permanência na educação superior: fatores socioeconômicos como condicionantes da evasão. Revista Cocar, 11(21), 66-89. https://periodicos.uepa.br/index.php/cocar/article/ view/1282

Santos, R. J. L. L., Sousa, E. P., Rodrigues, G. M. M., \& Quaresma, P. C. (2019). Estresse em acadêmicos de enfermagem: importância de identificar o agente estressor. Brazilian Journal of Health Review, 2(2), 1086-94. https://www. brazilianjournals.com/index.php/BJHR/article/ view/1304/1183

Vasconcelos, T. C., Dias, B. R. T., Andrade, L. R., Melo, G. F., Barbosa, L., \& Souza, E. (2015). Prevalência de sintomas de ansiedade e depressão em estudantes de medicina. Revista Brasileira de Educação Médica, 39(1), 135-142. http://dx.doi.org/10.1590/1981-52712015v39n1e00042014 